\title{
Formation of Neuronal Pathways in the Imaginal Discs of Drosophila melanogaster ${ }^{1}$
}

\author{
Y. N. JAN, ${ }^{2}$ A. GHYSEN, ${ }^{*}$ I. CHRISTOPH, S. BARBEL, AND L. Y. JAN \\ Howard Hughes Medical Inslitule and Department of Physiology, School of Medicine, Universily of California, San Francisco, \\ San Francisco, California 94143 and "Laboratoire de genetique, Universite libre de Bruxelles, 67 rue des Chevaux, 1640 Rhode-St- \\ Genese, Belgium
}

\begin{abstract}
We have followed the formation of neuronal pathways in different imaginal discs of Drosophila. The pattern is highly reproducible for a given disc type but distinct for each type of discs: in leg discs, several neurons are present before metamorphosis and provide two major pathways that are joined by later neurons; in the wing and haltere discs, a few pairs of neurons appear after the onset of metamorphosis and pioneer the major pathways; in antenna discs, no pioneers are detected before massive neuronal differentiation begins. The mechanisms used for axonal guidance seem common to all discs, and the differences between discs can be accounted for simply by differences in the arrangement and birth time of pioneer neurons. Different subsets of pioneer neurons are deleted by mutations such as scute and engrailed.
\end{abstract}

An essential property of nerve cells is their ability to send out axons and connect to specific target cells. In order to reach their targets, axons often have to travel over considerable distances. A good example is the case of insect sensory axons, which first have to travel from the peripherally located cell body to the central nervous system, and then to their precise target within the central nervous system. A crucial part of the mechanism that allows axons to reach distant targets may be their ability to follow pre-existing neuronal pathways, at the periphery (Wigglesworth, 1953) as well as in the central nervous system (Ghysen, 1978; Goodman et al., 1982). Consistent with this view is the fact that stereotyped "pioneer" pathways are set up in insect embryos, both peripherally (Bate, 1976) and centrally (Bate and Grunewald, 1981; Goodman et al., 1981). Pathfinding by subsequent neurons could then be accounted for by the "labeled pathway" hypothesis (Ghysen and Janson, 1980; Raper et al., 1983). According to this hypothesis, axonal pathways established by pioneer neurons are differentially marked, most likely on their surface, and growth cones of neurons that appear later are

Received November 26, 1984; Revised February 19, 1985;

Accepted February 19, 1985

\footnotetext{
${ }^{1}$ We are grateful to Mr. Larry Ackerman for electron microscopy and preparation of figures We wish to thank Dr. Mike Rate for communicating his findings on the embryonic origin of leg pioneer neurons before publication, Dr. Flora Katz for helpful comments on the manuscript, and Dr. Tom Kornberg for providing the engrailed stocks. This work is supported by National Institutes of Health Research Grant NS 19191 to L. Y. J. and Y.N. J., and a North Atlantic Treaty Organization grant to A. G., Y. N. J., and L. Y. J.

${ }^{2}$ To whom correspondence should be addressed.
}

differentially determined in their ability to recognize and follow a particular labeled pathway.

Recent studies on limbs of grasshopper embryos yielded much information on pathway formation by peripheral pioneer neurons (Bate, 1976; Ho and Goodman, 1982; Bentley and Caudy, 1983a, b; Keshishian and Bentley, 1983a, b, c; Berlot and Goodman, 1984). It has been suggested that pioneer growth cones rely on at least two sources of guidance: (1) polarity along the limb axis, and (2) strategically located cells that serve as stepping stones (Bate, 1976), guideposts (Bentley and Keshishian, 1982), or landmarks (Taghert et al., 1982) along the prospective pathway. However, the nature of the polarity signal and of the interaction between the growing axon and its stepping stones remains obscure. One way to test these hypotheses is to use a genetic approach which takes advantage of appropriate mutations as tools to interfere with the normal process. In addition, identifying genes with functions important in the process of pathway formation may eventually lead to a description of this process in molecular terms. Drosophila is an obvious choice for this type of analysis (Thomas and Wyman, 1982; Palka et al., 1983; Zipursky et al., 1984). One potential drawback of the fly is its small size, which might make it difficult to study pathway formation at the cellular level. However, the pioneering pathways for adult sensory axons are simple and highly invariant so that their formation is analyzable in details approaching those achieved in larger insects (C. M. Bate, personal communication; Murray et al., 1984) and mutations may specifically affect different components of a given pathway, as shown in this paper.

Another interest of working with Drosophila is related to a major difference in the development of grasshoppers and flies. Grasshoppers are hemimetabolous insects, in which the larva is essentially a miniature adult. Thus, most or all pathway formation occurs during embryogenesis, and postembryonic development involves mostly an increase in the number and size of sensory structures. By contrast, in holometabolous insects such as flies, the adult derives from nests of undifferentiated cells present in the larva (the imaginal discs), so that the adult sensory structures appear de novo during or shortly before metamorphosis. Thus, it would be of interest to know if the strategy used to set up highly precise pioneer pathways during the embryonic development holds in the very different case of the adult sensory axons in holometabolous insects. Here we report that the strategy used to set up the pathways differs not only between the fly and the grasshopper, but also between different discs and even between different classes of neurons within one disc. Our results suggest that the underlying mechanisms of axonal guidance may be common to all systems and that the impressive variety of strategies can be accounted for by differences in the location and birth time of pioneer neurons.

\section{Materiais and Methods}

Stocks. Drosophila melanogaster were raised on standard cornmeal-yeastagar medium at $25^{\circ} \pm 1^{\circ} \mathrm{C}$. Normal flies were of Oregon-R (OR) wild type 
strain. The $\operatorname{sc}^{10-1}$ flies were obtained from the California Institute of Technology Stock Center as sc ${ }^{10-1} / y$ Hw (Lindsley and Grell, 1968). The sc $^{10-1}$ larvae were identified as male animals with dark brown $\left(y^{+}\right)$mouth hooks. The $e n^{1}$ and $e n^{2}$ flies were kindly provided by $\mathrm{T}$. Kornberg at University of California, San Francisco.

Immunogen preparation and immunization. Homogenates of 7 - to 10 -hrold $\mathrm{OR}$ wild type embryos were used to immunize mice. About 1000 to 2000 embryos were homogenized in $0.5 \mathrm{ml}$ of Robb's medium ( $40 \mathrm{~mm} \mathrm{NaCl}, 40$ $\mathrm{mm} \mathrm{KCl}, 0.4 \mathrm{mM} \mathrm{KH}_{2} \mathrm{PO}_{4}, 0.4 \mathrm{mM} \mathrm{Na}_{2} \mathrm{HPO}_{4}, 1.2 \mathrm{mM} \mathrm{MgSO}_{4}, 1.2 \mathrm{mM} \mathrm{MgCl}$, $10 \mathrm{~mm}$ glucose, $1 \mathrm{~mm} \mathrm{CaCl}$, $\mathrm{pH} \mathrm{7.2)}$ with a glass homogenizer (Konte). The homogenate was added to $0.5 \mathrm{ml}$ of complete Freund's adjuvant and emulsified. Two female 6-week-old BALB/c mice were each injected intraperitoneally with $0.4 \mathrm{ml}$ of the emulsion. The animals were boosted intravenously (through the tail vein) 3 weeks later either with $0.4 \mathrm{ml}$ of embryo homogenate (4 to 500 embryos in Robb's medium) or with $0.4 \mathrm{ml}$ of homogenate of grasshopper embryonic central nervous system at $40 \%$ development (about 100 nervous systems). The latter scheme was done in collaboration with Kathryn Kotrla and Corey Goodman of Stanford University. The idea was to increase the chance of generating monoclonal antibodies that recognize both Drosophila and grasshopper nervous system, preferably ones that recognize homologous structures in the two species.

Hybridoma fusion and screening. The protocol used here is essentially that of Herzenberg et al. (1978). Three days after the last intravenous immurication, the mice were sacrificed and their spleens were dissected out. Speen lympocytes $\left(1.3 \times 10^{8}\right)$ were fused with SP2 myeloma cells $(1.3$ $\times 10^{8}$ ) by using $50 \%$ polyethelene giycol. The fused cells were put into 96 well plates containing HAT medium as selecting agent for hybridomas. About 10 days after fusion, supernatants from each well that had hybridoma growth were screened with immunofluorescence for useful antibodies. For this purpose, hundreds of embryos of mixed age ( 0 to $22 \mathrm{hr}$ ) were mixed with a drop of O.C.T. embedding medium (Miles Laboratories) and mounted on a cryostat specimen holder and quickly frozen. Sections $(12-\mu \mathrm{m})$ were cut with a SLEE cryostat. Ribbons of cryostat sections on glass slides were fixed for 30 min in Robb's medium containing $2 \%$ formaldehyde. The sections were then processed with indirect immunofluorescence as described below. Ilybridomas producing antibodies of interest were cloned by picking single cells and placing each into a well containing thymus feeder cells. Thus, cells that grow up in each well belong to a single clone. These wells were then rescreened with immunofluorescence. Altogether, more than 50 clones producing antibodies specific for subsets of neurons, muscle cells, extracellular matrix, and so on, were obtained.

Immunohistochemical staining of the imaginal discs. Because the imaginal discs are thin, immunocytochemistry can be done on whole mounts, without the necessity for sectioning and reconstruction. To isolate discs, third instar larvae or pupae were pinned on a Sylgard dish under Robb's medium. The animals were dissected open along the dorsal midline. The discs were then freed of their connections with the central nervous system and body wall. The staging of lavae and pupae were as described by Bodenstein (1950). Pupation begins when a larva becomes immobile, with their spiracles everted and white puparium forming. Timing of puparium formation is accurate within $1 \mathrm{hr}$.

For immunocytochemical staining, the discs were first treated for $10 \mathrm{~min}$ with $0.1 \mathrm{mg} / \mathrm{ml}$ of collagenase (Worthington) to remove the peripodial membrane and then fixed for 60 min at room temperature in Robb's medium containing $2 \%$ formaldehyde. The discs were then incubated overnight at $4^{\circ} \mathrm{C}$ in $0.1 \mathrm{M}$ phosphate buffer $(\mathrm{pH} 7.6)$ containing $1 \%$ normal horse serum and $0.3 \%$ Triton $X-100$. After washing, the discs were incubated with monoclonal antibody (MAb) 21A4 or other MAbs diluted 1:10 in $0.1 \mathrm{M}$ phosphate buffer containing $0.3 \%$ Triton X-100.

After the discs were washed for $1.5 \mathrm{hr}$ in phosphate buffer, they were then subjected to either immunofluorescence staining or immunoperoxidase staining; for immunofluorescence staining the discs were incubated for 1.5 $\mathrm{hr}$ at room temperature with fluorecein-conjugated goat anti-mouse IgG (Monoclonal Inc.) that was diluted 1:50 in phosphate buffer. After washing, the discs were mounted in glycerol:phosphate buffer (3:1) on slides and viewed with an epifluorescence microscope.

Immunofluorescence staining provided a quick way to view the neuronal pathways in the discs. However, when permanent records or better threedimensional structures of the discs were desired, immunoperoxidase staining was used instead. Briefly, the discs were incubated with biotinylated horse anti-mouse lgG, washed extensively, and then incubated with avidin-biotinylated horseradish peroxidase (HRP) conjugates. The treated discs were then subjected to the diaminobenzidine reaction according to the protocol of Ghysen (1978). After ethanol dehydration and clearing with xylene, the stained discs were mounted in Permount.
Pathway formation in each type of imaginal discs was followed at hourly intervals during the first $6 \mathrm{hr}$ of pupation. At least 10 samples were examined at each stage. The patterns observed have been rather consistent among different discs.

Electron microscopy. The imaginal discs were dissected out in $2 \%$ glutaraldehyde, $2 \%$ paratormaldehyde in $0.1 \mathrm{~m}$ phosphate buffer, $\mathrm{pH} 7.2$. The tissue was left in the fixative overnight, rinsed in phosphate buffer, and then postfixed with $1 \%$ osmium tetroxide in the same buffer. Dehydration was started with $35 \%$ ethanol followed by a brief rinse in $70 \%$ ethanol, and then en bloc staining with $2 \%$ uranyl magnesium acetate in $70 \%$ ethanol for 3 to $18 \mathrm{hr}$. Dehydration was completed with $95 \%$ and $100 \%$ ethanol. The discs were then embedded in Epon 812 epoxy. Very pale gold sections were stained with $1 \%$ aqueous uranyl acetate and lead citrate. The specimens were examined with a JEOL $100 \mathrm{~B}$ or a Philips 400 electron microscope.

\section{Results}

An MAb recognizes peripheral nervous system in Drosophila. In order to follow the neural development of Drosophila, we have produced a number of MAbs showing specificity for Drosophila nervous system (Jan and Jan, 1981). One of the MAbs, 21A4, is a good neuronal marker. All peripheral neurons appear to contain this antigen because (1) in all of the tissues that we have examined, including the antennae, eyes, halteres, and body wall muscles, MAb $21 \mathrm{~A} 4$ stains all known sensory neurons as well as motor axons, and (2) the same neurons in the peripheral nervous system were stained when antibodies specific for HRP were used instead of MAb 21A4; previous studies have shown that the antiperoxidase antibodies bind to all neuronal membranes in Drosophila (Jan and Jan, 1982) and possibly in grasshoppers as well (Jan and Jan, 1982; Bentley and Caudy 1983a, b; Keshishian and Bentley, 1983a, b, c). Immunocytochemical studies at the electron microscopic level showed that the antigen recognized by MAb 21A4 is intracellular. Western blots indicate that this antigen is a polypeptide of $M_{r}=200,000$ (data not shown). Compared to antiperoxidase antibodies, MAb 21A4 gives a stronger signal and lower background, and serves as a supcrior neuronal marker.

Using this antibody we searched the Drosophila nervous system for simple and easily analyzable neuronal pathways. After examining embryos, larvae, early pupae, and adult flies, we concentrated on two interesting stages. The first one is the development of the early peripheral nervous system in the embryo ( 9 to $13 \mathrm{hr}$ after fertilization) A detailed description of this study will be published elsewhere $(E$ Aceves, Y. N. Jan and L. Y. Jan, manuscript in preparation). The second system is the development of pathways in the imaginal discs, which we will describe here.

Similar and complementary information concerning development of peripheral neurons in the leg disc and the wing disc has also been obtained by $C$. M. Bate (personal communication) and Murray et al. (1984), respectively, using antibodies against HRP.

The leg discs. Imaginal discs are essentially hollow sacs composed of a single layer of epithelium plus other associated cells. In late third instar larvae, the leg discs are flat and have a clearly concentric organization with the future most distal segment; i.e., the future tip of the leg, situated in the center, whereas more proximal leg segments are derived from more peripheral annuli (Schubiger 1968). This folded epithelium has two faces. The apical face will secrete the cuticle and become the outer face of the adult leg, whereas the basal face will secrete the basal lamina and become the internal face of the epidermis. As the larva pupates, each leg disc unfolds and extends out from the apical face like a telescope to form the tubular pupal leg. The appearance and further development of neurons can be followed by immunocytochemical staining using the 21A4 antibody. Figure 1 shows an example of the neuronal staining in a pupal leg disc.

In late third instar larvae, seven neurons are already present in each leg disc. Four neurons (A1, A2, A3, and A4) will form the anterior pathway. Two other neurons ( $P 1$ and $P 2$ ) will form the posterior pathway, and a solitary neuron $(F)$ is present in the future femur segment (Fig. 2). Of the anterior group, the cell bodies of $A 1$, 


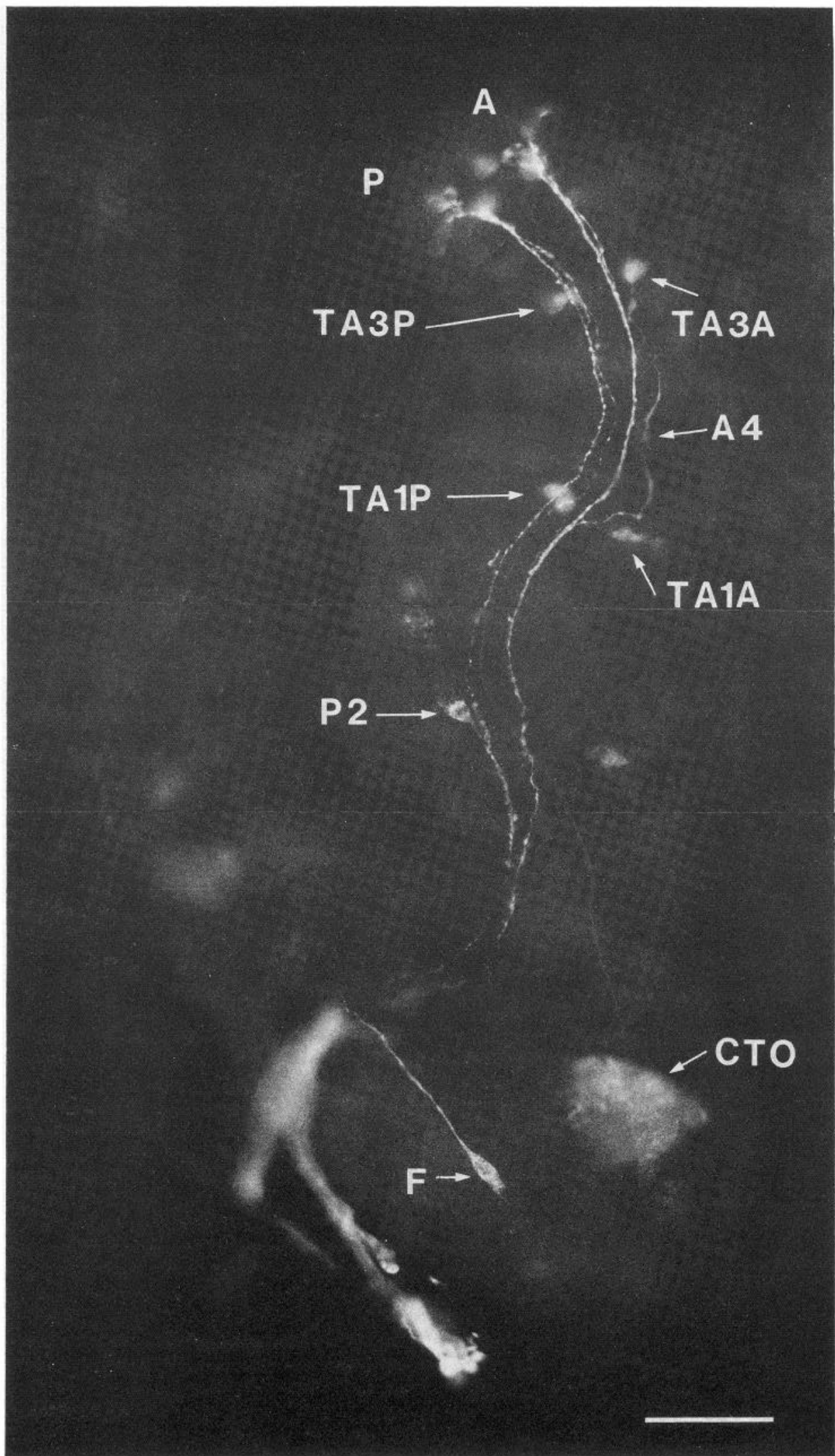

Figure 1. Neuronal pathways in a leg disc $5 \mathrm{hr}$ APF. The disc was stained with immunofluorescence using MAb 21A4. Some of the cells are out of the focal plane. $A$, anterior; $P$, posterior. TA1P indicates a cell in the anterior-posterior pathway at the first tarsal seg. ment. TA3A and TA3P indicate neurons in the third tarsal segment. $A 4$ and $P 2$ are larval pioneer neurons (see the text). Bar, $50 \mu \mathrm{m}$.

$A 2$, and $A 3$ are positioned near the center of the disc and the cell body of A4 is located more anteriorly; all four cell bodies are quite close to the apical face of the disc. Of the posterior group, the cell body of P1 is positioned near the center of the disc, close to the apical face, whereas the cell body of P2 is close to the basal face of the disc. Each of these neurons sends out its axon across the thickness of the disc to the basal face, where they join a larval nerve that runs along the disc and connects it to the central nervous system. Thus, late larval leg discs already contain axonal pathways. These pathways are established early during embryogenesis (C. M. 
(A) 3rd I

(B) $0-1 \mathrm{~h}$ APF

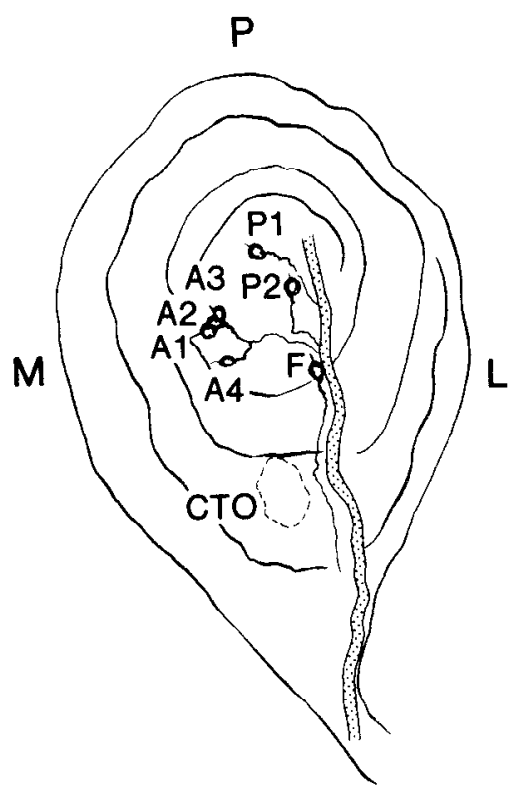

A

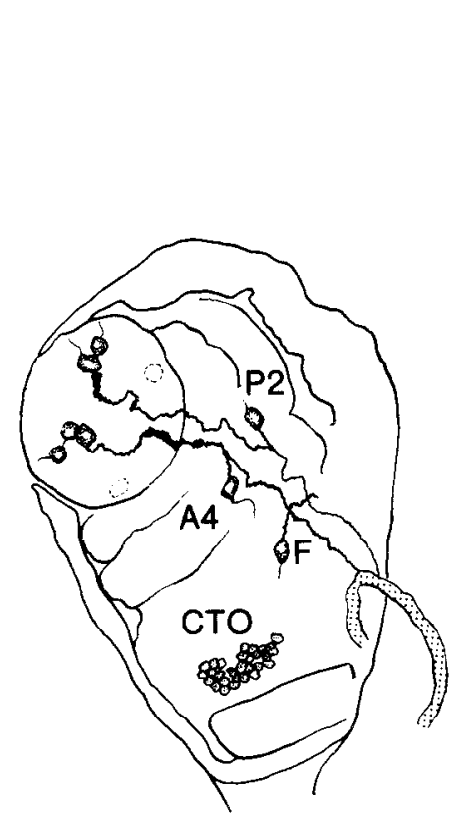

Figure 2. Development of neuronal pathways in the leg disc. Camera lucida tracings of discs stained with immunoperoxidase using MAb $21 \mathrm{A4}$ are shown for the following developmental stages: $A$, late third instar larval stage; $B, 1 \mathrm{hr}$ APF; $C, 4 \mathrm{hr}$ APF. $A$, anterior; $P$, posterior; $M$, medial; $L$, lateral; $F e$, femur; $T i$, tibia; TAN, Nth segment of tarsus. Bar, $50 \mu \mathrm{m}$.

Bate, personal communication). At the onset of metamorphosis, the discs start to evaginate. The cell bodies at the center of the disc, $A 1, A 2, A 3$, and $P 1$, move with the future tip of the leg whereas their axons simply elongate. By $1 \mathrm{hr}$ after puparium formation (APF) (Fig. $2 B$ ), the leg begins to assume a tubular shape; these four neurons are now located at the very tip of the leg and the two pathways formed by their axons run side by side along the length of the leg down to the base. A4 joins the anterior pathway at the level of the second tarsal segment, and P2 joins the posterior pathway at the level of the tibia segment. At this time new neurons start to appear near the tip.

During the next few hours APF, the disc elongates rapidly. Simultaneous with the elongation, several groups of neurons differentiate and pioneer a secondary branching pattern. Figure $2 \mathrm{C}$ shows the pathways in leg discs at $4 \mathrm{hr}$ APF. By this time, the leg disc has already assumed a tubular shape and individual segments are clearly visible. The most prominent secondary branches at this time are in the first and third tarsal segments (TA1 and TA3). In each segment, a pair of large neurons (TA1A, TA1P; TA3A, TA3P) form two side branches, one joining the anterior pathway and one joining the posterior pathway (Figs. $1 C$ and $2 \mathrm{C}$ ).

In addition to these two pairs of neurons, several other neurons are now visible. From the tip to the base, they are: (i) several neurons in the tip (tarsal 5 segment, TA5); (ii) two pairs of neurons in tarsal 4 segment (TA4); (iii) a pair of neurons in the tibia segment, (Ti); (iv) a single very large neuron $(F)$ in the femoral segment (Fe; this is probably the seventh larval neuron); and ( $v$ ) several neurons at the base of the everted leg, possibly in the trochanter and coxal segments. By $6 \mathrm{hr}$ APF these neurons have axons growing toward but not yet reaching the two major pathways.
(C) $4 \mathrm{~h}$ APF

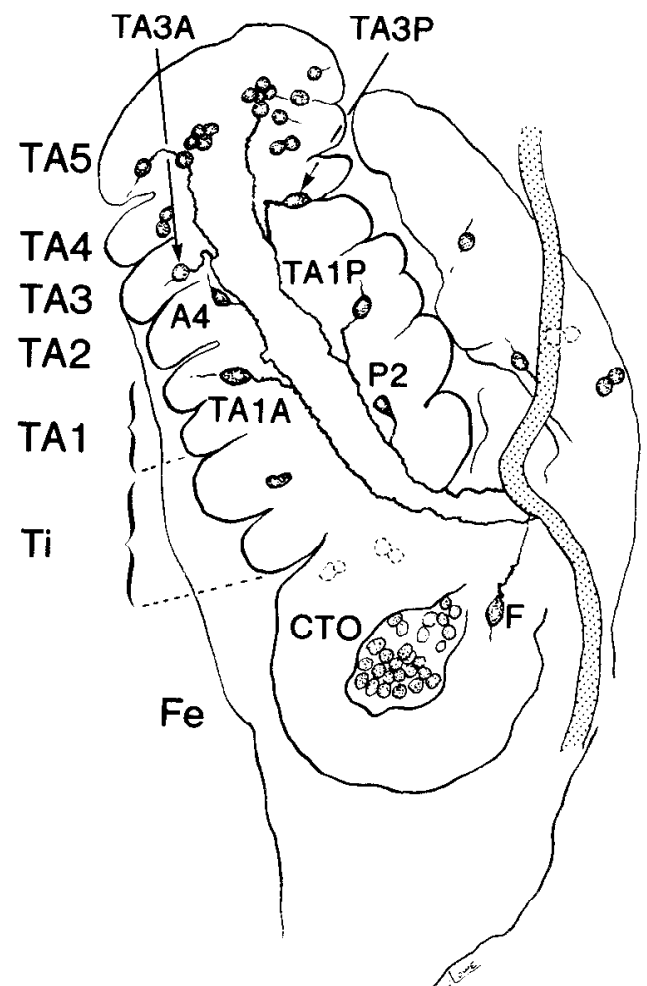

In addition to the neurons described above, there is a large cluster of more than 20 neurons in the femur that will become the cordotonal organ (CTO). In late third instar larvae these neurons are barely visible and show no axonal outgrowth. Those neurons start to differentiate at the time of puparium formation. At $4 \mathrm{hr}$ APF (Fig. $3 A$ ), their axons extend toward the base of the leg. By 6 hr APF they have reached and jointed the major pathways (Fig. $3 B$ ).

Information used in the formation of these secondary pathways resides within the leg discs. No signs of motor axons arriving from the central nervous system were detected throughout this period. Furthermore, leg discs dissected from third instar larvae can be induced to evaginate in vitro (Siegel and Fristrom, 1978). In cultured leg discs, which are free from any central influence, pathway formation proceeds normally ( $A$. Jones and $Y$. N. Jan, unpublished results). The same was found to be true for pathway formation in wing discs (Edwards et al., 1978; Blair et al., 1984).

In summary, seven neurons are present in the late larval imaginal disc. These neurons are responsible for the presence of two pathways that run the entire length of the everting leg. Within the first few hours APF, a new set of neurons differentiate and set up a highly reproducible pattern of branches to the two early pathways. Notable features of the pathway formation are; first, the two early pathways that extend from the tip to the base of the everted leg are established without the "pioneer axons" having to find their way along the rapidly extending leg. Second, the axons that set up the branches have to travel only a relatively short distance before joining one of the early pathways. Third, before a developing axon reaches one of the early pathways, its growth is clearly oriented. This is particularly obvious in the case of the massive CTO bundle growing out of the large femoral cluster (Fig. 3, $A$ and $B$ ). 

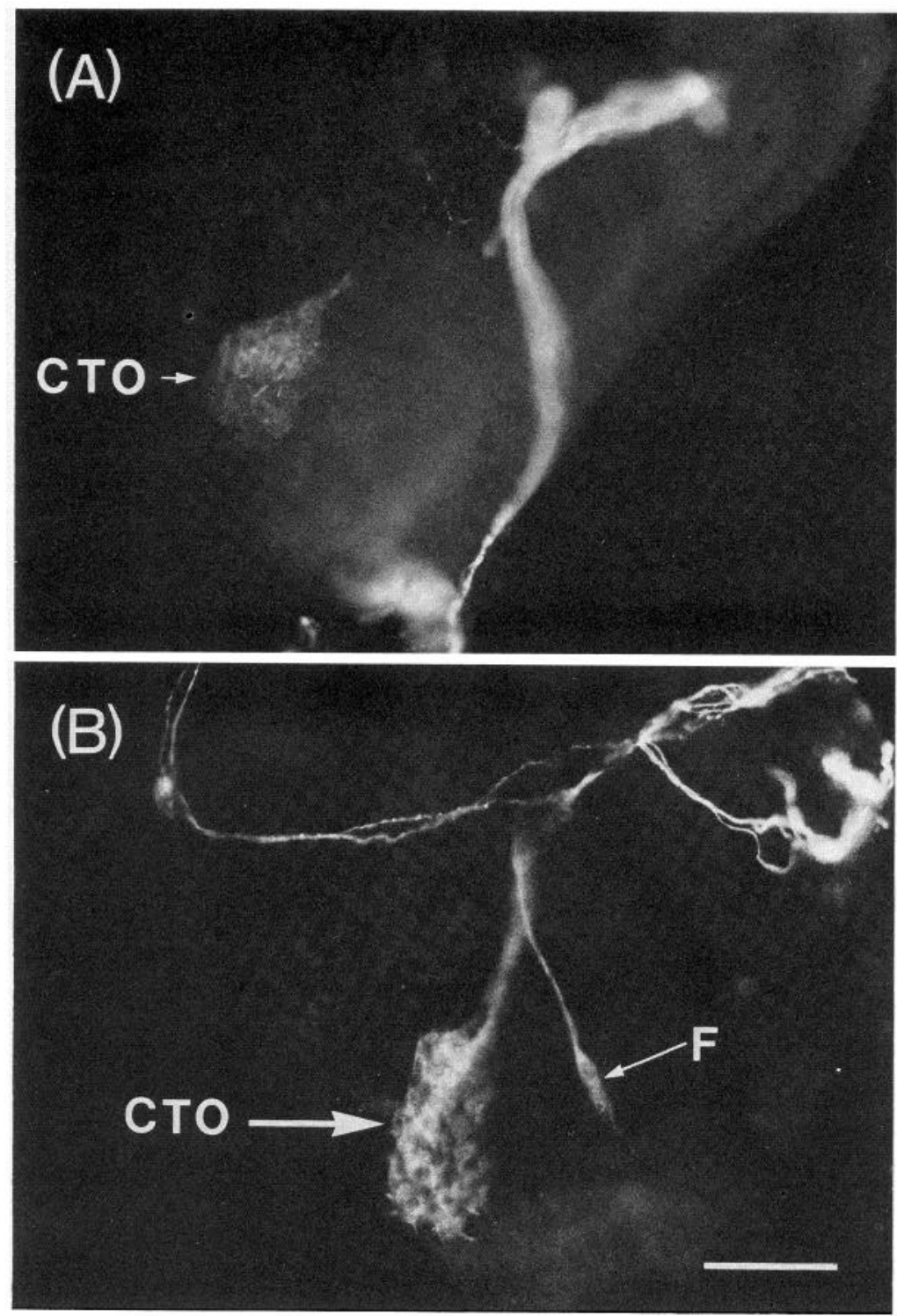

Figure 3. Development of the CTO. The leg discs were stained with immunofluorescence using MAb 21A4. A, Four hours APF; $B, 6 \mathrm{hr}$ APF. Notice the highly oriented axonal outgrowth from the CTO toward the point where all major neuronal pathways in the leg converge. Bar, $50 \mu \mathrm{m}$.
The wing disc. Unlike the leg discs, the wing discs of third instar larvae contain no neurons. Within the first hour APF, however, several neurons appear almost simultaneously in different regions of the wing disc. We have not analyzed in detail the many small neurons that differentiate in the region of the disc that will become the notum, and we will briefly describe only neurons that appear in the wing itself, because similar, independent observations have been made by Murray et al. (1984) using antibodies to HRP.

Very soon after the onset of metamorphosis, the wing disc everts and its central region unfolds to form an elongated pouch that will eventually become the wing blade. In the distal part of the everted wing pouch, three pairs of neurons differentiate within $1 \mathrm{hr}$ APF. One pair, the TSM(1) and the E-T neurons (Murray et al. 1984), appears near the anterior edge whereas the other two pairs are located in the middle of the wing disc (Fig. $4 A$ ). The proximal pair corresponds to the $\mathrm{ACV}$ and the $\mathrm{E}-1$ neurons, whereas the distal pair corresponds to the L3-2 and the E-2 neurons in Murray et al. (1984). In the more proximal region of the pouch, which will form the wing base and hinge, a string of large neurons appears in the middle of the disc ( $\mathrm{vHCV}$ ) and a single pair appears near the anterior edge (dHCV) (Murray et al., 1984). During the next few hours, all of these neurons send out axons proximally (Fig. $4 B$ ), so that by $5 \mathrm{hr}$ APF the processes from the distal pair, L3-2 and E-2, reach the cell bodies of the proximal pair, ACV and E-1, while the axons of the latter join the vHCV cluster. Thus, one major pathway of the wing blade is established. This pathway corresponds to the nerve bundle along vein L3 in the adult (Murray et al., 1984). Meanwhile, the processes from TSM(1) and E-T have pioneered a second pathway, corresponding to the bundle along vein L1 in the adult (Murray et al., 1984). The paths along veins $L 1$ and $L 3$ have been shown to provide unique guidance cues to supernumerary neurons that appear later in the wings of certain mutants (Palka et al., 1983).

Shortly after the appearance of these neurons, rows of neurons differentiate along the entire anterior edge of the wing (Fig. 4); these neurons correspond to the rows of sensory bristles of the anterior margin (triple and double rows). They begin to send out axons 6 to $7 \mathrm{hr}$ APF.

The haltere disc. The haltere is the metathoracic counterpart of the wing. However, the distal part of the haltere (the capitellum) has little sensory innervation. The proximal part and hinge, in contrast, are massively innervated.

Like wing discs, haltere discs of late larvae have no neurons. Shortly after the onset of metamorphosis, a clear pathway defined by 10 to 15 neurons is formed in the future base and hinge region (Fig. $5 A$ ); this cluster bears some resemblance to the vHCV cluster of the wing. A second cluster of neurons appears in the most proximal (thoracic) region of the disc (not shown). No neuron is observed in the distal region up to $7 \mathrm{hr}$ APF. 

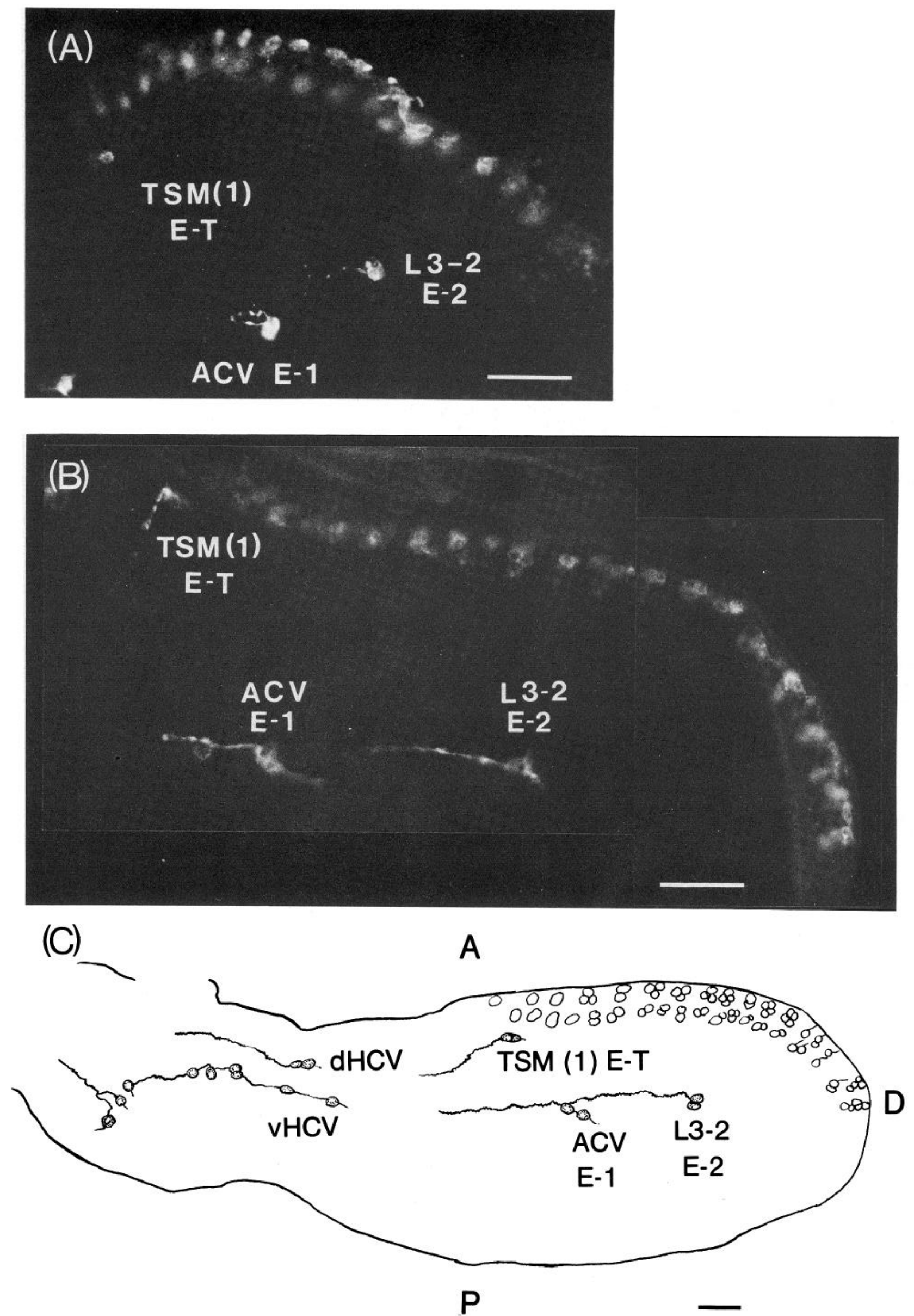

Figure 4. Development of neuronal pathways in wing discs. A, Four hours APF; $B, 6 \mathrm{hr}$ APF-immunofluorescence staining of discs; $C$, a Camera lucida tracing of a disc (6 hr APF) stained with immunoperoxidase using MAb 21A4. A, anterior; $P$, posterior; $D$, distal. TSM(1), E-T, ACV, E-1, L3-2, E-2, vHCV, and $\mathrm{dHCV}$ are wing pioneer neurons (see the text). Bar, $50 \mu \mathrm{m}$. 

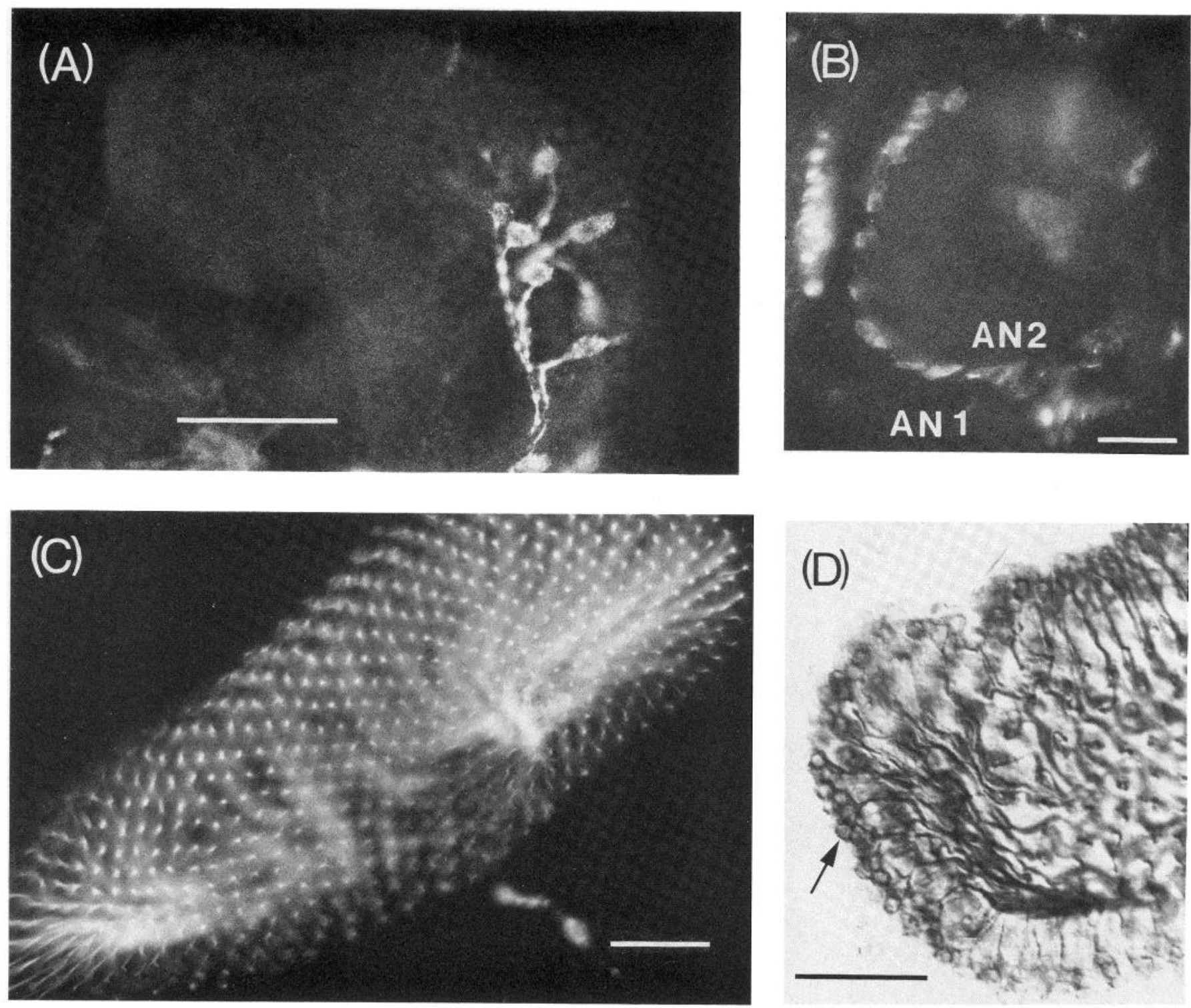

Figure 5. Neuronal pathways in imaginal discs. A, A haltere disc, $6 \mathrm{hr}$ APF; $B$, an antenna disc, $6 \mathrm{hr}$ APF, $C$ and $D$, Eye discs, $3 \mathrm{hr}$ APF. $A$ to $C$ show immunofluorescence staining; $D$ shows immunoperoxidase staining. MAb $21 \mathrm{~A} 4$ was used in all cases. The arrow in $D$ points to developing photoreceptor cells and their axons. $A N 1$ and $A N 2$, first and second anterior segment. Bar, $50 \mu \mathrm{m}$.

The eye-antenna disc. The posterior, cup-shaped part of this disc will develop into the eye and part of the cephalic capsule. Its major neuronal components are the photoreceptor cells in the retina. During the third larval instar, a wave of differentiation sweeps anterionwards across the eye disc, leaving in its wake a lattice of highly ordered clusters of cells, the future ommatidia (CamposOrtega and Gateff, 1976; Ready et al., 1976). The photoreceptors in these clusters are labeled by the antibody in late larval discs (Fig. $5, C$ and $D$ ). The axons from old clusters have already joined the main bundle to the optic lobes of the brain, whereas younger clusters just behind the differentiation wave are just beginning to send out their axons.

The anterior (antennal) part of the eye-antenna disc has a concentric organization very similar to that of a leg disc. Here again, the innermost region will give the most distal structures of the adult antenna, and the surrounding annuli will give more proximal segments. Unlike the leg disc, however, the antennal disc does not elongate very much after the onset of metamorphosis. Moreover, no neurons are observed in the central region of late larval antenna discs. Not until $6 \mathrm{hr}$ APF have two concentric rings of neurons appeared (Fig. 5B), probably corresponding to the richly innervated first and second antennal segments. Thus, it appears that even though antennae and legs are serially homologous appendages, the formation of neuronal pathways involves different strategies in the two cases.

Early neuronal pathways in $s c^{10-1}$ discs. The achaete-scute region near the tip of the $X$ chromosome contains a set of genes involved in neurogenesis (Garcia-Bellido and Santamaria, 1978; Jimenez and Campos-Ortega, 1979; White, 1980). Mutants that lack all of these genes have no central nervous system left except in the head. This defect seems to result from degeneration of neuroblasts soon after they segregate from the embryonic ectoderm (Jimenez and Campos-Ortega, 1979). Mutations in one of these genes, scute, affect mostly the adult sensory structures. An extreme allele, $s c^{10-1}$, eliminates virtually all bristles from the adult body. The larval sensory structures, however, appear unaffected by this mutation.

Immunocytochemical studies of leg discs of $s c^{10-1}$ larvae show no obvious abnormality. After the onset of metamorphosis, the discs evert normally. The seven larval neurons are brought to the tip of the disc, and the two early pathways running from the tip to the 

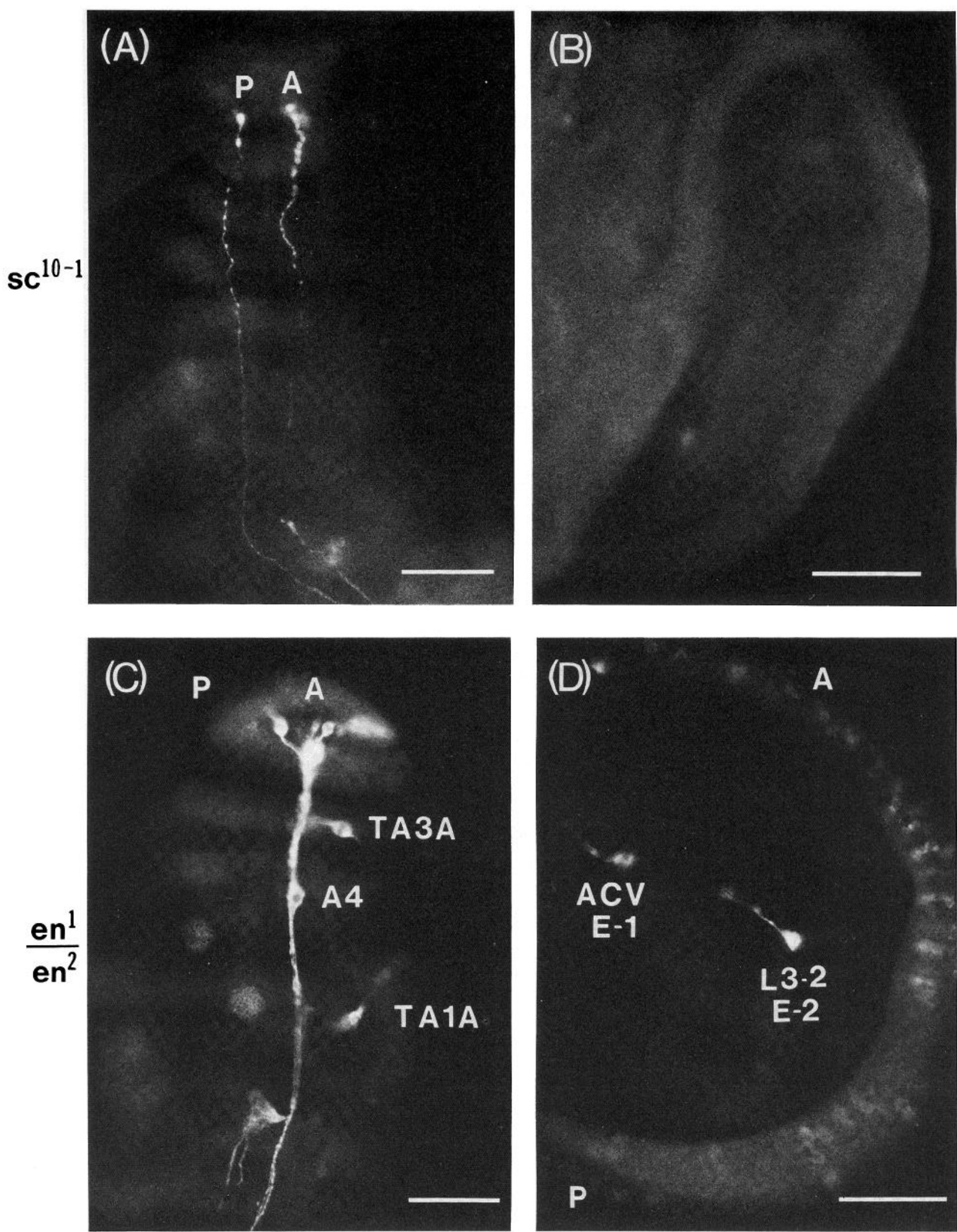

Figure 6. Abnormal neuronal pathways in the imaginal discs of $s c^{10-1}$ and $e n^{1} / e n^{2}$ pupae. Immunofluorescence staining with MAb 21A4. A, An $s c^{10-1}$ leg disc, $4 \mathrm{hr}$ APF. Notice the lack of pupal pioneers as compared with wild type discs shown in Figures 1 and 2. B, An $s c^{10-1}$ wing disc, 4 hr APF. Notice the absence of any pioneer neurons. $C$, An $e n^{1} / e n^{2}$ leg disc, $4 \mathrm{hr}$ APF. Notice that only the anterior pathway is present. $D$, An en $1 / e n^{2}$ wing disc, $5 \mathrm{hr}$ APF. Notice that the wing margin neurons were duplicated in part of the posterior compartment. $A$, anterior; $P$, posterior. A4, TA1A, ACV, E-1, L3-2, and E-2 are pioneer neurons (see the text). Bar, $50 \mu \mathrm{m}$. 

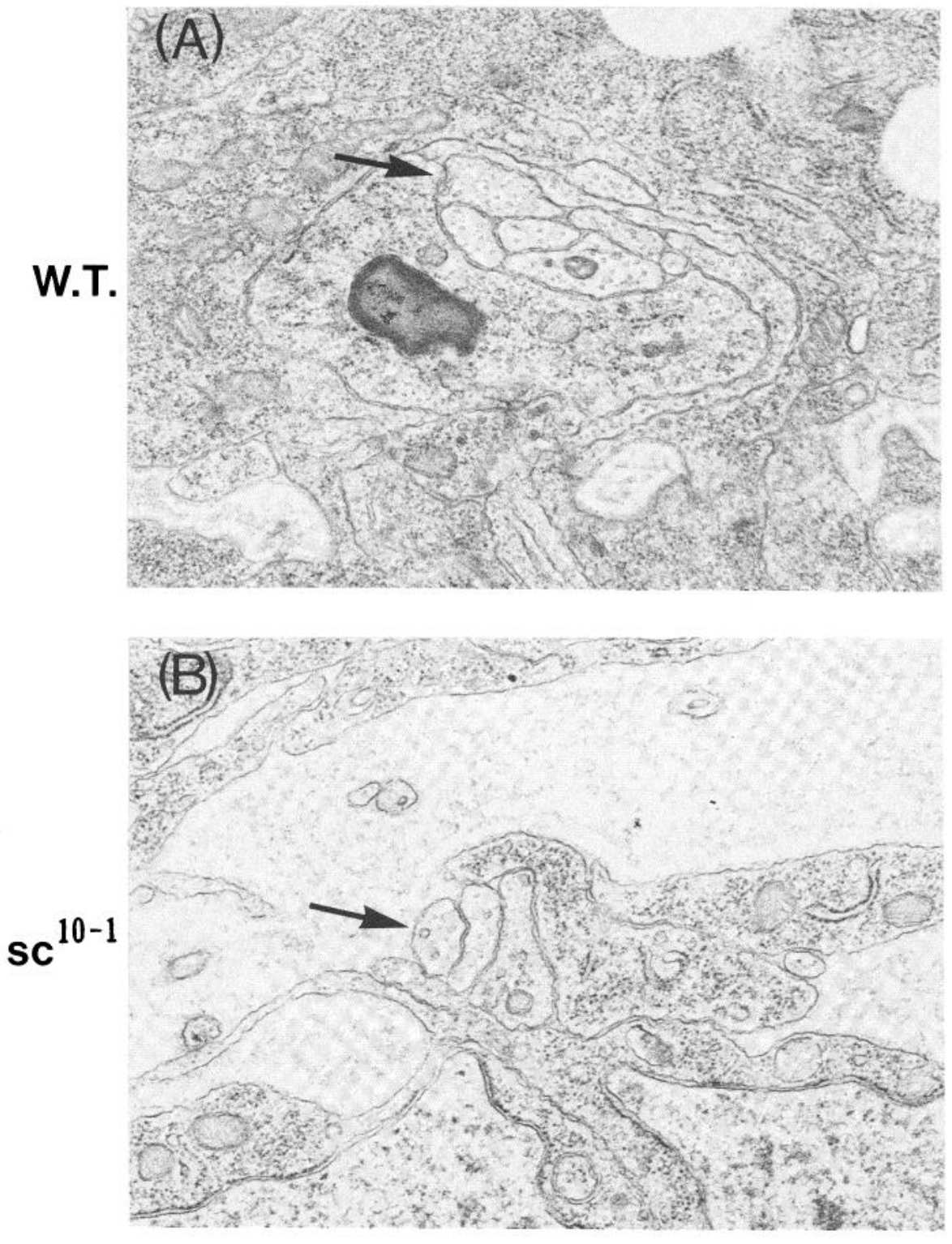

Figure 7. Electron micrographs of the anterior pathway in leg discs, $4 \mathrm{hr}$ APF. The sections were taken at the level of TA3 (third tarsal segment). A, A wild type (W.T.) OR disc. Notice that eight axons in the cross-section are present. $B$, An $s c^{10-1}$ disc. Notice that only three axons are present. C. An en'/ $e n^{2}$ disc. The normal number of axons is present in the anterior pathway. In this $e n^{1} / e n^{2}$ disc, the posterior pathway is completely missing (not shown). Arrows point to the axon bundles. Bar, $0.5 \mu \mathrm{m}$.

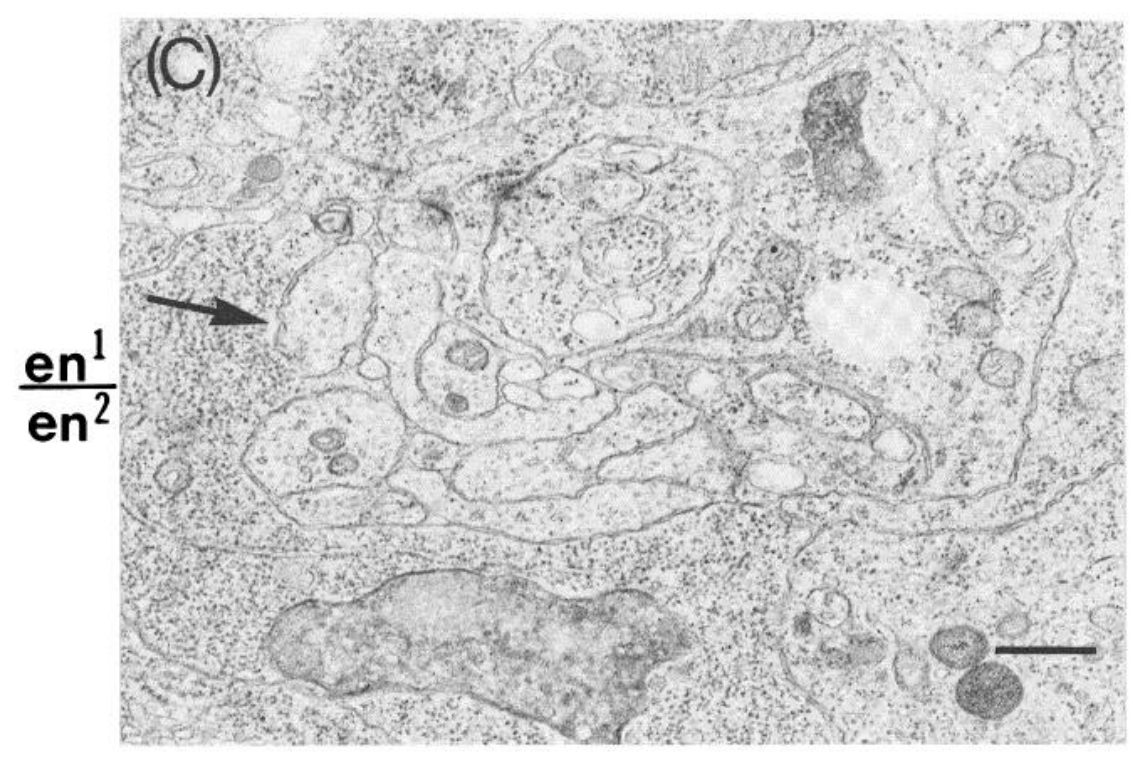


base are formed much as in wild type flies. However, the pupal pioneers which should appear at this stage and form the branching pattern never appear (Fig. 6A). The possibility that these neurons did appear but were not recognized by the 21A4 antibody is ruled out by the following observations: (1) the same defect is observed when anti-HRP was used instead of MAb 21A4 (not shown); (2) electron microscopic studies of sections of everted leg discs at the level of TA3 show that, in $S^{10-1}$, there are only three axons in the anterior pathway and one axon in the posterior pathway, whereas about eight axons are present each in normal legs at the same stage (Fig. 7). It is possible that the pupal neurons in $s c^{10-1}$ discs appear noimally but die before sending out their axons, as suggested for the central neurons in embryos with large achaet-scute deficiencies (Jimenez and Campos-Ortega, 19/9). The defect in pupal pioneer differentiation is not limited to the leg discs: in wing discs, neither the wing blade pioneers nor the triple and double row neurons appear up to $7 \mathrm{hr}$ APF (Fig. 6B)

Early neuronal pathways in engrailed leg discs. The gene engrailed is responsible for the formation of anterior-posterior compartments in all segments (Garcia-Bellido and Santamaria, 1972; Garcia-Bellido et al., 1973; Morata and Lawrence, 1975; Kornberg, 1981; Lawrence and Struhl, 1982; Epper and Sanchez, 1983). Mutations in this gene affect only cells in the posterior compartments, making them resemble their anterior counterparts to a variable extent. Since in leg discs there are two distinct neuronal pathways, one anterior and one posterior, we wondered whether en mutations might selectively affect the posterior pathway. For this purpose, we used $e n^{1} / e n^{2}$, a genotype that yields viable flies with a strong engrailed phenotype.

We studied the wing and leg discs of $e n^{1} / e n^{2}$ larvae and early pupae. In wing discs, some of the structures along the anterior edge (the wing margin neurons) are duplicated along the posterior edge (Fig. 6D). However, the situation in the leg disc is quite different. In greater than $90 \%$ of the mutant leg discs, the posterior pathway is completely missing, whereas the anterior one looks normal and includes the cluster of small neurons in TA5 and TA4, the major branches in TA3 and TA1, and the A2 neurons (Fig. 6C). In about $10 \%$ of the cases, the posterior pathway was missing but the pupal neurons of that pathway were present (not shown). Although some of these neurons were not far from the anterior pathway, they did not send out their processes to join the anterior pathway.

The absence of the posterior pathway in $e n^{1} / e n^{2}$ discs is not due to the absence of the 21A4 antigen in the posterior neurons; electron microscopic analyses showed that the posterior pathway is indeed absent in pupal legs $6 \mathrm{hr}$ APF.

\section{Discussion}

A simple system to analyze neuronal pathway formation. Neuronal pathway formation in the imaginal discs of Drosophila is simple and highly accessible to experimentation. The imaginal discs appear as a favorable system for studying pathway formation for the following reasons. (1) Much is already known about the development of imaginal discs (reviewed in Bryant, 1978). (2) The neuronal pathways in each type of imaginal disc are highly invariant (for wing disc, see also Murray et al., 1984). Their main features are established withirı a few hours APF by a small number of neurons which appear at precise locations at precise times. (3) The early neurons are only slightly smaller than the well-studied pioneers in the grasshopper embryo, and many can be identified with interference optics in unstained, living preparations. This makes it possible to use techniques such as intracellular dye injections to follow the growth of individual neurons and their processes. (4) The development of pathways proceeds normally when the discs are cultured in vitro (Edwards et al. 1978; Blair et al., 1984; A. Jones and Y. N. Jan, unpublished data). Thus, one can experimentally manipulate the early events of pathway formation by selective cell killing or by exposure to various agents. (5) One can use pathway formation in imaginal discs as an assay system to analyze the effects of mutations that alter neuronal development or function.

Mechanisms of axonal guidance. Superficially, the pattern of pathway formation appears to ditfer in different discs, yet the underlying mechanisms of axonal guidance need not be very diverse. At least two sources of guidance that were reported to orient the growth of grasshopper pioneer axons (Bentley and Caudy, 1983a, b; Berlot and Goodman, 1984) seem to be involved in pathway formation in Drosophila as well. The first one is the strong tendency of axons to join other neurons and to fasciculate with other axons, as seen most clearly in the wing and leg pathways. This property has been observed in the grasshopper for both periphera and central pioneers (Bentley and Keshishian, 1982; Raper et al., 1983), and in many other systems as well. In the peripheral pioneer system, this property appears crucial because it allows a growing axon to use strategically located neurons as stepping stones, or to join already existing pathways, in its journey toward the central nervous system.

A second source of guidance is the tendency of axons to respond to polarity cues (Nardi, 1983; Bentley and Caudy, 1983a, b; Berlot and Goodman, 1984). In most discs we have observed neurons growing in an oriented manner for more than $100 \mu \mathrm{m}$ before coming into contact with other neurons, axons or any detectable landmark (e.g., the wing pioneers, and the leg CTO cluster). Blair et al. (1984) have cultured distal fragments of wing discs and found that the distal L $3-2$ and E-2 neurons still grew proximally even in the absence of the proximal $A C V$ and $E-1$ neurons. Since the neuronal pathways form normally in discs induced to evaginate in vitro (Edwards et al. 1978; Blair et al., 1984; A. Jones and Y. N. Jan, unpublished data), the polarity cues must reside within the disc and could be provided by epidermal cells or by the basal lamina they produce. In the moth, grafting experiments suggest that polarity cues reside in the epidermis (Nardi and Kafatos, 1976; Nardi, 1983). To test whether growing axons respond to epidermally distributed polarity cues, it would be interesting to analyze the effect on axonal orientation of mutations that alter the polarity pattern of epidermal cells (Grubb and GarciaBellido, 1982).

Strategies of pathway formation. Different strategies of pathway formation are used in different discs. This is rather surprising, considering that some discs represent homologous structures in different parts of the body (e.g., legs and antenna) and can be transformed into each other by homoeotic mutations (Postlethwait and Schneiderman, 1971). The leg discs are unique in that they appear to use larval neurons as "guiding neurons." These larval neurons are formed early during embryogenesis and their dendrites innervate Keilin organs that are present in the epidermis of each thoracic segment (C. M. Bate, personal communication). The wing discs have no such larval guides. At the onset of metamorphosis, several pairs of pioneers appear simultaneously (see also Murray et al., 1984). The case of the antenna disc is different again. Here no pioneers are detected even after the onset of metamorphosis, up to the time when a large number of neurons appear and differentiate simultaneously. The grasshopper limb shows yet another pattern. In this case, different segments of the pathway are pioneered sequentially so that the final tip-to-base path is obtained in a stepwise fashion.

Why is there such apparent diversity in the strategies used for pathway formation? Considering guidepost cells and polarity as two possible mechanisms of pathway formation, we think that differences in the strategies used may simply reflect different problems these neurons face in finding their ways to the central nervous system from the various peripheral structures. The leg disc is the one that undergoes the most drastic configuration change during evagination. Within the first $6 \mathrm{hr}$ APF, the distance from the tip to the base increases 10-fold, from about $40 \mu \mathrm{m}$ to more than $400 \mu \mathrm{m}$. Thus, a pioneer born after the onset of metamorphosis would be faced with rapidly increasing distances and a rapidly changing topography. However, the larval pioneers, simply by having their cell 
bodies located at the center of the disc, will provide a tip-to-base pathway without any need to navigate their growth cones in a rapidly increasing distance of changing terrain. The neurons that appear subsequently need only to navigate a distance of no more than 30 to $50 \mu \mathrm{m}$ in an already evaginated disc before they join a major pathway. In the grasshopper, filopodia of growing neurons are found to extend over a distance of $50 \mu \mathrm{m}$ (Taghert et al., 1982).

The situation is different in the wing disc, where the conformation change is much more modest during the first $6 \mathrm{hr}$ APF. By the time the pioneers differentiate, the disc is completely everted but the distances involved are much smaller than in the leg disc. The pioneers are located in such a way that proximally growing processes of distal neurons will come in contact with proximal neurons and thereby form a major pathway. The margirn neurons, which appear slightly later, are so densely packed that the pathway could consist of the cell bodies themselves.

In the antennal disc, eversion is very slow and the distances remain very short at all times. Thus, there is no obvious need for an elaburale guiding system, and the absence of distinct pioneer fibers before massive neuronal differentiation may not be too surprising.

Unlike the Drosophila appendages, which evert without much cell division, the limbs of the grasshopper grow mostly by cell proliferation. The mitotic activity is most intense at the tip of the limb bud. Thus, the different limb segments are formed sequentially from proximal to distal, rather than being formed simultaneously by the unfolding of an already complete sheet of cells. In each segment of the grasshopper limb, the pathway is set up by a few pairs of pioneer neurons located in such a way that proximally oriented growth will bring the axon close to a more proximal pair of ncurons, much as in the case of Drosophila wing pioneers. As development proceeds and another segment is formed, another stretch of the pathway may be added to the already existing, more proximal stretch.

Thus in cach casc, the strategy used to form a pathway appears well adapted to the particular developmental modality. It is remarkable that the same few mechanisms of axonal guidance appear to be used in the different discs, and in the grasshopper limb as well. An extreme variety in strategies need not involve the acquisition of new mechanisms. Rather, this variety can be ascribed specifically to the location and time of appearance of neurons in the developing appendage.

Effects of mutations on pioneer neurons. The analysis of pathway formation in mutant flies shows that mutations may affect specific subsets of pioneers. In the leg disc, for example, two main pathways are set up by early (larval) pioneers which are unaffected by the scute mutation, whereas the branches to the two main pathways are pioneered by late (pupal or adult) neurons, which are completely suppressed by $s c^{10-1}$. In contrast, in en leg discs one pathway (the posterior one), is suppressed, including both early and late pioneers, whereas the other is left intact. These results suggest the existence of differences in the genetic control among the set of neurons that pioneer the leg pathway.

The reason the posterior pathway should often disappear in en discs, rather than being transformed to its anterior counterpart, is not known. Similar deletion, rather than duplication, of structures has been found in en genital discs (Epper and Sanchez, 1983). Clonal analysis has shown that the frequency of en clones in posterior compartments is abnormally low (Lawrence and Struhl, 1982), indicating that many of them are lost during development. It may be that cell lethality results from the apposition of cells with conflicting determinations. The absence of axonal outgrowth from posterior neurons, or of these neurons themselves, suggests that pioneer neurons either express or detect compartment-specific properties. This is unexpected since it has been shown that sensory axons from different compartments, or even from different segments, can fasciculate together (Lawrence, 1975; Ghysen and Deak, 1978; Palka et al., 1981). It would be interesting to see whether differences in compartmental determination might influence other functions of the pioneer axons, such as their guiding cues within the central nervous system.

Neuronal pathway formation in imaginal discs is simple and can be analyzed at the single-cell level. This may provide an assay system in the search for mutations that specifically affect neuronal pathway formation. The very existence of $s c$ and en mutations, that specifically affect parts of the neuronal pathway in imaginal discs, indicates that one may be able to isolate mutations that affect the primary mechanisms in pathway formation (e.g., polarity cues or their reading, neuron recognition, or fasciculation). By studying the corresponding genes, one may hope to learn about the molecular basis of pathway formation.

\section{References}

Bate, C. M. (1976) Pioneer neurons in an insect embryo. Nature 260: 5455.

Bate, C. M., and E. B. Grunewald (1981) Embryogenesis of an insect nervous system. II. A second class of neuron precursor cells and the origin of the intersegmental connectives. J. Embryol. Exp. Morphol. 61: 317-330.

Bentley, D., and M. Caudy (1983a) Navigational substrates for peripheral pioneer growth cones: Limb axis polarity cucs, limb scgment boundaries and guidepost neurons. Cold Spring Harbor Symp. Quant. Biol. 48: 573585 .

Beritley, D., and M. Caudy (1983b) Pioneer axons lose directed growth after selective killing of guidepost cells. Nature 304: 62-65.

Bentley, D., and $H$. Keshishian (1982) Pathfinding by peripheral pioneer neurons in grasshoppers. Science 218: 1082-1088.

Berlot, J., and C. S. Goodman (1984) Guidance of peripheral pioneer neurons in the grasshopper: Adhesive hierarchy of epithelial and neuronal surfaces. Science 223: 493-496.

Blair, S. S., L. M. Nagy, and J. Palka (1984) Axon guidance in surgically manipulated wing discs of Drosophila. Neurosci. Abstr. 10: 139.

Bodenstein, D. (1950) The postembryonic development of Drosophila. In Biology of Drosophila, M. Demerec, ed., pp. 275-367, Hafner Publishing Co., New York.

Bryant, P. J. (1978) Pattern formation in imaginal discs. In The Genetics and Biology of Drosophila, Volume 2C, M. Ashburner and T. R. F. Wright, eds., pp. 229-335, Academic Press, Inc., New York.

Campos-Ortega, J. A., and E. A. Gateff (1976) The development of the ommatidial patterning in metamorphosed eye imaginal discs implants of Drosophila melanogaster. W. Roux Arch 179: 373-392.

Edwards, J. S., M. J. Milner, and S. W. Chen (1978) Integument and sensory nerve differentiation of Drosophila imaginal discs in vitro. W. Roux Arch. 185: $59-77$

Epper, F., and L. Sánchez (1983) Effects of engrailed in the genital disc of Drosophila melanogaster. Dev. Biol. 100: 387-398.

Garcia-Bellido, A., and P. Santamaria (1972) Developmental analysis of the wing disc in the mutant engrailed of Drosophila melanogaster. Genetics 72: $87-104$

Garcia-Bellido, A., and P. Santamaria (1978) Developmental analysis of the achaete-scute system of Drosophila melanogaster. Genetics 88: 469 486.

Garcia-Bellido, A., P. Ripoll, and G. Morata (1973) Developmental compartmentalization of the wing disk of Drosophila. Nature 245: 251-253.

Ghysen, A. (1978) Sensory neurons recognise defined pathways in Drosophila central nervous system. Nature 274: 869-872.

Ghysen, A., and I. I. Deak (1978) Experimental analysis of sensory nerve pathways in Drosophila. W. Roux Arch 184: 273-283.

Ghysen, A., and R. Janson (1980) Sensory pathways in Drosophila central nervous system. In Development and Neurobiology of Drosophila $O$. Siddigi, P. Babu, L. M. Hall, and J. C. Hall, eds., pp. 247-265, Plenum Publishing Co., New York.

Goodman, C. S., C. M. Bate, and N. C. Spitzer (1981) Embryonic development of identified neurons: Origin and transformation of the $\mathrm{H}$ cell. J. Neurosci. 1: 94-102.

Goodman, C. S., J. A. Raper, R. K. Ho, and S. Chang (1982) Pathfinding by neuronal growth cones during grasshoppers embryogenisis. Symp. Soc. Dev. Biol. 40: 275-316.

Grubb, D., and A. Garcia-Bellido (1982) A genetic analysis of the determination of cuticular polarity during development in Drosophila melanogaster. J. Embryol. Exp. Morphol. 68: 37-57.

Herzenberg, L. A., L. A. Herzenberg, and C. Milstein (1978) Cell hybrids of myelomas with antibody forming cells and T-lymphomas with T cells. In 
Handbook of Experimental Immunology, D. M. Weir ed., pp. 25.1-25.7, Blackwell, London.

Ho, R. K., and C. S. Goodman (1982) Peripheral pathways are pioneered by an array of central and periphera neurons in grasshopper embryos. Nature 297: 404-406

Jan, L. Y., and Y. N. Jan (1981) Neuropharmacology of insects. Ciba Found. Symp. 88: 221-239.

Jan, L. Y., and Y. N. Jan (1982) Antibodies to horseradish peroxidase as specific neuronal markers in Drosophila and in grasshopper embryos. Proc. Natl. Acad. Sci. U. S. A. 79: 2700-2704

Jimenez, F., and J. A. Campos-Ortega (1979) On a region of Drosophila genome necessary for central nervous system development. Nature 282 310-312

Keshishian, H., and D. Bentley (1983a) Embryogenesis of peripheral nerve pathways in grasstopper legs. I. The initial nerve pathway to the CNS. Dev. Biol. 96: 89-102.

Keshishian, H., and D. Bentley (1983b) Embryogenesis of peripheral nerve pathways in grasshopper legs. II. The major nerve routes. Dev. Biol. 96 : $103-115$.

Keshishian, H., and D. Bentley (1983c) Embryogenesis of peripheral nerve pathways in grasshopper legs. III. Development without pioneer neurons. Dev. Biol. 96: 116-124.

Kornberg, T. (1981) Compartments in the abdomen of Drosophila and the role of the engrailed locus. Dev, Biol. 86: 363-372.

Lawrence, P. A. (1975) The structure and properties of a compartment border: The intersegmental boundary in Oncopeltus. Ciba Found. Symp. 29: 3-23.

Lawrence, P. A., and G. Struhl (1982) Further studies of the engrailed phenotype in Drosophila. EMBO J. 1:827-833.

Lindsley, D. L., and E. H. Grell (1968) Genetic variations of Drosophila melanogaster. Carnegie Institution, Washington, D. C.

Morata, G., and P. Lawrence (1975) Control of compartmental development by the engrailed gene in Drosophila. Nature 255: 614-617.

Murray, M. A., M. Schubiger, and J. Palka (1984) Neuron differentiation and axon growth in the developing wing of Drosophila melanogaster. Dev. Biol. 104: 259-273.
Nardi, J. B. (1983) Neuronal pathfinding in developing wings of the moth Manduca sexta. Dev. Biol. 95: 163-174.

Nardi, J. B., and F. C. Kafatos (1976) Polarity and gradients in lepidopteran wing epidermis. II. The differential adhesiveness model: Gradient of a nondiffusible cell surface parameter. J. Embryol. Exp. Morphol. 36: 489512

Palka, J., M. Schubiger, and H. S. Hart (1981) The path of axons in Drosophila wings in relation to compartment boundaries. Nature 294: 447-449.

Palka, J., M. Schubiger, and R. L. Ellison (1983) The polarity of axon growth in the wing of Drosophila melanogaster. Dev. Biol. 98: 481-492.

Postlethwait, J. H., and H. A. Schneiderman (1971) Pattern formation and determination in the antenna of the homoeotic mutant Antennapedia of Drosophila melanogaster. Dev. Biol. 25: 606-640.

Raper, J. A., and M. Bastiani, and C. S. Goodman (1983) Pathfinding by neuronal growth cones in grasshopper embryos. II. Selective fasciculation onto specific axonal pathways. J. Neurosci. 3: 31-41.

Ready, D. F., T. F. Hanson, and S. Benzer (1976) Development of the Drosophila retina, a neurocrystalline lattice. Dev. Biol. 53: 217-240.

Schubiger, G. (1968) Anlageplan, Determinationszustand und Transdeterminationsleistungen der mannlichen Vorderbeinscheibe von Drosophila melanogaster. W. Roux Arch. 160: 9-40.

Siegel, J. G., and J. W. Fristrom (1978) The biochemistry of imaginal disc development. In The Genetics and Biology of Drosophila, M. Ashburner and T. R. F. Wright, eds., Vol. 2a, pp. 317-394, Academic Press, Inc. New York.

Taghert, P. H., M. J. Bastiani, R. K. Ho, and C. S. Goodman (1982) Guidance of pioneer growth cones: Filopodial contacts and coupling revealed with an antibody to Lucifer Yellow. Dev. Biol. 94: 391-399.

Thomas, J. B., and R. J. Wyman (1982) A mutation in Drosophila alters normal connectivity between two identified neurons. Nature 298: 650651

White, K. (1980) Defective neural development in Drosophila melamogaster embryos deficient for the tip of the $Y$ chromosome. Dev. Biol. 80: $332-$ 344.

Wigglesworth, V. B. (1953) The origin of sensory neurons in an insect. Q. J. Microsc. Sci. 94 : 93-112.

Zipursky, S. L., T. R. Venkatesh, D. B. Teplow, and S. Benzer (1984) Neuronal development in Drosophila retina: Monoclonal antibodies as molecular probes. Cell 36: 15-26. 